\title{
Standardisierte in-vitro-Modelle zur Charakterisierung von Stoßwellen
}

\author{
Franz Brümmer, Dierk Suhr und Dieter F. Hülser
}

Universität Stuttgart, Biologisches Insitut, Abt. Biophysik, Pfaffenwaldring 57, D-7000 Stuttgart 80

Bereits 1970 konnte durch in vitro Versuche gezeigt werden, daß sich Nierensteine mit Stoßwellen, welche sich in Wasser ausbreiten, zerkleinern lassen /11/. In den folgenden Jahren wurde ein System entwickelt (UnterwasserFunkenstrecke) /10/, mit dem 1980 die ersten Nierensteine durch extrakorporale Stoßwellenlithotripsie erfolgreich behandelt wurden /5/. In der Zwischenzeit ist dieses Verfahren zu einer Standardmethode in der Harnsteintherapie geworden. Verschiedenartigste Untersuchungen haben jedoch gezeigt, daß die nicht invasive Stoßwellenlithotripsie auch mit den neueren Geräten der zweiten und dritten Generation (elektromagnetische und piezoelektrische Stoßwellenerzeugung) nicht frei von Nebenwirkungen ist. So wurden neben intra- und perirenalen Hämatomen und Ödemen auch arterielle Hypertonie und Funktionsstörungen der Niere festgestellt (Übersicht: $/ 4 / 6 / 13 /$ ). Zwischenzeitlich wird die Stoßwellenlithotripsie auch bei Gallenblasensteinen /19/ und Speichelsteinen /12/ erfolgreich eingesetzt. Eine umfassende Charakterisierung der biologischen Stoßwellenwirkung ist daher dringend erforderlich, insbesondere da es zu Mehrfachbehandlungen infolge erfolgloser Erstbehandlung /16/ oder Rezidivfällen kommt.

Die physikalische Charakterisierung von fokussierten Unterwasser-Stoßwellen wurde schon beschrieben /7/15/. Die Ergebnisse zeigen jedoch starke Schwankungen, nicht nur, wenn man Hydrophone unterschiedlicher Hersteller vergleicht, sondern auch bei verschiedenen Hydrophonen einer Baureihe /7/8/. Darüber hinaus werden die bisher zur Verfügung stehenden Hydrophone durch die Stoßwellen sehr schnell beschädigt. Hier können neue Entwicklungen wie kapazitive Hydrophone /9/ oder optische Sondenhydrophone /21/ vielleicht Abhilfe schaffen. Zum jetzigen Zeitpunkt ist die physikalische Vermessung von Stoßwellen allerdings noch nicht problemlos möglich. Außerdem ist unbekannt, welche Stoßwellenparameter (wie Anstiegszeit und Dauer der Über- und Unterdruckamplitude) für die Steinzertrümmerung und die biologischen Nebenwirkungen bedeutsam sind. Wir haben deshalb vier in-vitro-Modelle standardisiert, mit denen sich Wirkungen von Variationen der Behandlungsparameter schnell und zuverlässig aufzeigen lassen. Weiterhin kann die Stoßwellenwirkung auf Zellen, Gewebe und Steine in Lithotriptern mit unterschiedlichen Stoßwellenquellen getestet und verglichen werden (Review: /14/17/).

1. L1210 Einzelzellsuspension: Zellen der Maus-LeukämieZellinie L1210, die als Suspensionskultur wachsen, werden in Polyethylenpipetten überführt und mit Stoßwellen behandelt. Danach wird mit einem elektronischen Zellzählgerät der Anteil der intakten Zellen bestimmt. Nach Anfärbung mit zwei Farbstoffen (Fluoresceindiacetat, Propidiumiodid) kann der Anteil der lebenden und toten
Zellen in einem Durchflußzytometer gemessen werden. Lebende Zellen sind in der Lage, Fluoresceindiacetat, welches in die Zelle diffundiert, zum fluoreszierenden Fluorescein zu hydrolysieren. Zellen, deren Membran für den Ausschlußfarbstoff Propidiumiodid permeabel ist, gelten als physiologisch tot. Aus den erhaltenen DosisEffekt-Kurven wird die $\mathrm{LD}_{\mathrm{s}_{0}}$ berechnet $/ 2 /$.

2. Multizell-Sphäroide: Hierbei handelt es sich um dreidimensionale Zellaggregate, die aus Zellen unterschiedlichster Art und Herkunft gezüchtet werden können. Multizell-Sphäroide stellen eine Annäherung an die Situation in vivo dar, da im Inneren, wo sich die Zellen in einem selbstgeschaffenen Mikromilieu befinden, Zell-Zell-Kontakte und extrazelluläre Matrix wie im Gewebe ausgebildet werden. Mit den herkömmlichen Verfahren der licht- und elektronenmikroskopischen Histologie lassen sich die Auswirkungen der Stoßwellen beim Durchdringen der Aggregate lokalisieren und darstellen.

Nach Immobilisierung der Einzelzellen und Multizell-Sphäroide lassen sich Primär- und Sekundäreffekte der Stoßwellenwirkung unterscheiden $/ 1 /$.

3. Humane Enythrozyten: Menschliche Erythrozyten aus verfallenen Blutkonserven werden nach zweimaligem Waschen in phosphatgepufferter Salzlösung in Polyethylenpipetten gefüllt und Stoßwellen ausgesetzt. Danach kann der Hämoglobingehalt im Überstand photometrisch gemessen und die $\mathrm{LD}_{s 0}$ berechnet werden.

4. Modellsteine: Steine aus einem Zahnzement/Glaskügelchen-Gemisch (artificial hmt stones, High-MedicalTechnologies, Oberach, Schweiz; Durchmesser $15 \mathrm{~mm}$, Gewicht 1,45-1,53 g) werden Stoßwellen ausgesetzt, bis sie in Fragmente kleiner als $2 \mathrm{~mm}$ zertrümmert sind. Die dazu benötigte Stowellenanzahl dient als $\mathrm{Ma} B$ für die Zertrümmerungswirkung. Diese Kunststeine sind von der Deutschen Elektrotechnischen Komission im DIN und VDE (DKE) als Prüfkörper für Geräte zur extrakorporalen Lithotripsie empfohlen.

Mit diesen standardisierten in-vitro-Modellen läBt sich der Einfluß verschiedener Parameter wie z.B. Temperatur und Gasgehalt des Lithotripterbades, Position des Wasserspiegels, Generatorspannung, Elektrodenzustand, Umgebungsmedium usw. auf die Stoßwellenwirkung untersuchen. Weiterhin ist die Möglichkeit gegeben, verschiedene Lithotriptertypen zu vergleichen. So zeigten sich Unterschiede zwischen einem piezokeramischen Wandler und einem Gerät mit Unterwasser-Funkenstrecke /18/, die mit deren unterschiedlicher Fokusgeometrie zu erkären sind. Außerdem ist nach Variation oben genannter Para- 
meter eine Korrelation zwischen biologischer Wirkung und gleichzeitig gemessenen physikalischen Größen gefunden worden $/ 3 /$.

Die genannten Modelle haben unterschiedliche Vorund Nachteile. L1210-Zellen liefern gleichzeitig gemessene Aussagen über Zellschädigung, Membranpermeabilisierung und intrazelluläre Enzymaktivität. Dazu muß allerdings ein Zellkulturlabor vor Ort zur Verfügung stehen. Auch ohne diese Einrichtung kann die Messung durchgeführt werden, wenn die Zellen in einem vitalen Zustand angeliefert werden. Die kombinierte L1210-Methode ist dem TrypanblauAuschluB-Test überlegen: sie berücksichtigt auch den Anteil der zerstörten Zellen, der stets wesentlich höher ist als der Anteil der Zellen, deren Membran für Farbstoffe (Trypanblau oder Propidiumiodid) durchlässig geworden ist. Steht kein Durchflußzytometer zur Verfügung, so kann mittels eines Zellzählers, so wie er in jeder Klinik vorhanden ist, immer noch die Zellzerstörung bestimmt werden. Das L1210-Modell liefert dann immer noch quantitative Ergebnisse in Form von Dosis-Effekt-Kurven.

Anders verhält es sich mit den Multizell-Sphäroiden. Hier erhält man nur qualitative Ergebnisse, das Ausmaß der Schäden muß geschätzt werden. Darüber hinaus ist das Anfertigen der histologischen Präparate und deren Durchsicht sehr zeitaufwendig. Das Modell bietet aber den Vorteil, daß man den genauen Ort von Schäden lokalisieren kann - lichtmikroskopisch innerhalb des gewebeähnlichen Sphäroids, elektronenmikroskopisch sogar innerhalb einzelner Zellen.

Denkbar einfach zu handhaben sind die beiden letzten Modelle. Die Bestimmung des freigesetzten Hämoglobins ist mit jedem einfachen Photometer möglich, die Erythrozyten sind gekühlt uneingeschränkt transportabel. Nachteilig ist hier, $\mathrm{da} ß$ man nicht unterscheiden kann zwischen freigesetztem Hämoglobin aus zerstörten Zellen und solchem, welches durch transient permeabilisierte Membranen ansonsten intakter Erythrozyten austreten konnte. Dieses Modell erhält jedoch dadurch seine besondere Berechtigung, daß eine Zerstörung von Erythrozyten und Freisetzung von Hämoglobin auch in vivo bei Stoßwellenbehandlungen auftritt $/ 20 /$.

Die Kunststeine bieten die Möglichkeit, solche Parameter zu erkennen, die vorwiegend die Steinzertrümmerung beeinflußen und weniger biologische Effekte (Nebenwirkungen) hervorrufen. Damit ist die Möglichkeit gegeben, durch genaues Ausmessen der Anstiegszeit und Dauer der Über- und Unterdruckamplituden Aussagen über die Mechanismen der gewünschten bzw. unerwünschten Wirkungen zu bekommen.

Die hier vorgestellten transportablen Testsysteme eignen sich wegen ihrer Reproduzierbarkeit gut als Modelle, mit denen vergleichende Untersuchungen unterschiedlich generierter Stoßwellen durchgeführt werden können. Eventuell ließe sich mit ihnen sogar ein Teil der Tierversuche ersparen, die bislang noch nach jeder Modifikation an einem Stoßwellengerät durchgeführt werden.

\section{Literatur:}

/1/ Bräuner Th, Brümmer F, Hülser DF (1989) Histopathology of shock wave treated tumor cell suspensions and multicellular tumor spheroids. Ultrasound in Med. \& Biol. 15, 451-460.

/2/ Brümmer F, Brenner J, Bräuner Th, Hülser DF (1989) Effect of shock waves on suspended and immobilized L1210 cells. Ultrasound in Med. \& Biol. 15, 229-239.
/3/ Brümmer F, Staudenraus J, Nesper M, Suhr D, Eisenmenger W, Hülser DF (1989) Biological effects and physical characterization of shock waves generated by an XL-1 experimental lithotripter.

Proc. Ultrasonics International 89, 1130-1135.

14/ Brümmer F, Bräuner Th, Hülser DF (1990) Biological effects of shock waves. World J. Urology, in press.

/5/ Chaussy Ch (Hrg.) (1986) Extracorporeal shock wave lithotripsy: technical concept, experimental research and clinical application. Basel, S. Karger.

/6/ Chaussy Ch, Fuchs GJ (1989) Current state and future developments of noninvasive treatment of human urinary stones with extracorporeal shock wave lithotripsy. J. Urol. 141, 782-789.

/7/ Coleman AJ, Saunders JE (1989) A survey of the acoustic output of commercial extracorporeal shock wave lithotripters. Ultrasound in Med. \& Biol. 15, 213 227.

/8/ Coleman AJ, Saunders JE (1990) A comparison of PVdF hydrophone measurements in the acoustic field of a shock wave source. In press.

/9/ Filipczyński L, Etienne J (1990) Capacitance hydrophones for pressure determination in lithotripters. Ultrasound in Med. \& Biol. 16, 157-165.

/10/Forßmann B, Hepp W, Chaussy Ch, Eisenberger F, Wanner K (1977) Eine Methode zur berührungsfreien Zertrümmerung von Nierensteinen durch Stoßwellen. Biomedizinische Technik 22, 164-168.

/11/Häußler E, Kiefer W (1971) Anregung von Stoßwellen in Flüssigkeiten durch Hochgeschwindigkeitsregentropfen. Verh. Dtsche. Physikal. Gesell. 6, 786.

/12/Iro H, Meier J, Nitsche N, Wirtz PM, Ell Ch (1989) Extrakorporale piezoelektrische Lithotripsie von Speichelsteinen. HNO 37, 365-368.

/13/Lingeman JE, McAteer JA, Kempson SA, Evan AP (1988) Bioeffects of extracorporeal shock-wave lithotripsy. Urol. Clin. N. Am. 15, 507-514.

/14/Lubock P (1989) The physics and mechanics of lithotripters. Dig. Dis. Sci. 34, 999-1005.

/15/Müller M, Platte M (1985) Einsatz einer breitbandigen Piezodrucksonde auf PVDF-Basis zur Untersuchung konvergierender Stoßwellen in Wasser. Acustica 58, 215-222.

/16/Rassweiler J, Westhauser A, Bub P, Eisenberger F (1988) Second-generation lithotripters: a comparative study. J. Endourol. 2, 193-204.

/17/Reichenberger H (1988) Lithotripter systems. Proc. IEEE 76, 1236-1246.

/18/Riedlinger RE, Brümmer F, Hülser DF (1989) Pulsed high-power-sonication of concrements, cancer cells and rodent-tumors in vivo. Proc. Ultrasonics International 89, 305-312.

/19/Sauerbruch T, Delius M, Paumgartner G, Holl J, Wess O, Weber W, Hepp W, Brendel W (1986) Fragmentation of gallstones by extracorporeal shock waves. N. Engl. J. Med. 314, 818-822.

/20/Di Silverio F, Gallucci M, Gambardella P, Alpi G, Benedetti R, La Mancusa R, Pulcinelli FM, Romiti R, Gazzaniga PP (1990) Blood cellular and biochemical changes after extracorporeal shock wave lithotripsy. Urol. Res. 18, 49-51.

/21/Staudenraus J, Eisenmenger W (1988) Optisches Sondenhydrophon. Fortschritte der Akustik, DAGA '88, DPG GmbH, 467-470. 